\title{
Características físico-químicas de embutido curado fermentado com adição de carne de avestruz associada à de suíno
}

\author{
Physico-chemical characteristics of fermented cured sausage with the addition of ostrich meat \\ associated to pork meat
}

\author{
Carlos Pasqualin Cavalheiro ${ }^{\text {I }}$ Nelcindo Nascimento Terra ${ }^{\text {II }}$ Leadir Lucy Martins Fries ${ }^{\text {II }}$ \\ Liana Inês Guidolin Milani ${ }^{\mathrm{III}}$ Ana Paula de Souza Rezer ${ }^{\mathrm{III}}$ Caroline Viegas Cavalheiro $^{\mathrm{IV}}$ \\ Marialene Manfiov
}

\section{RESUMO}

Os objetivos deste trabalho foram desenvolver $e$ determinar as características físico-químicas e a composição centesimal de um embutido curado fermentado contendo carne de avestruz (Struthio camelus) e carne suína. Para isso, utilizaram-se quatro formulações, e em uma não foi utilizada carne de avestruz (controle) e nas outras foram utilizados 19, 38,3 e 57,6\% dessa carne, em associação com a carne suína. Os produtos foram avaliados durante o processamento diariamente, na primeira semana e nos dias 14, 21 e 28, para análise de $\mathrm{pH}$; nos dias zero, três, sete, 14, 21 e 28 foram avaliados quanto à atividade de água; e, após concluída a fabricação, foram avaliados quanto à quebra de peso e à composição centesimal (28 dias). Os resultados mostraram que os embutidos apresentaram um pH mais ácido do que o ideal. Peças com maior quantidade de carne de avestruz apresentaram uma maior perda de peso ao final do processamento, sendo a diferença entre o tratamento 3 e o tratamento controle de $8,2 \%$. O teor de carne de avestruz alterou significativamente as peças em relação ao teor de umidade final. Com exceção do nível de gordura, todos os outros quesitos avaliados atendem ao Regulamento Técnico de Identidade e Qualidade do Ministério da Agricultura.

Palavras-chave: embutido curado fermentado, carne de avestruz, $p H$, composição centesimal.

\section{ABSTRACT}

The aim of this research was to develop and to determine the physic-chemical characteristics and centesimal composition of a fermented cured sausage containing ostrich meat (Struthio camelus) and pork meat. Four different formulations were developed: one with no ostrich meat (control) and others with levels of $19,38.3$ and $57.8 \%$, respectively, in association with pork meat. The products were evaluated daily in the first week and in days 14, 21 and 28 for $p H$ analysis and days zero, three, seven, 14, 21 and 28 for water activity analysis. After manufacturing was completed, weight loss and centesimal composition (28 days) were evaluated. The results showed that the sausages presented $\mathrm{pH}$ levels more acidic than ideal. Products with greater quantity of ostrich meat presented greater weight loss at the end of procedure, in which the difference between treatment 3 and control was $8.2 \%$. Different levels of ostrich meat in the recipe altered the proximate analysis results significantly, especially in relation to the final moisture levels. With the exception of fat level, all the other attributes evaluated comply with the Technical Regulation of Identity and Quality of the Ministry of Agriculture.

Key words: cured fermented sausages, ostrich meat, $\mathrm{pH}$, centesimal composition.

\section{INTRODUÇÃO}

A estrutiocultura brasileira foi iniciada há 10 anos com a importação de matrizes da África do Sul e dos Estados Unidos. Atualmente, o Brasil possui o segundo maior rebanho de avestruzes (MUNIZ, 2006), porém o consumo dessa carne ainda é reduzido por conta de seu alto valor de mercado (BANI et al., 2007). Segundo MUNIZ (2008), pode-se estimar que, no ano de 2007, houve um consumo per capita de carne de

ICurso de Medicina Veterinária, Centro de Ciências Rurais (CCR), Universidade Federal de Santa Maria (UFSM), 97105-900, Santa Maria, RS, Brasil. E-mail: cavalheiro.carlos@hotmail.com. Autor para correspondência.

IDepartamento de Tecnologia e Ciência dos Alimentos (DTCA), CCR, UFSM, Santa Maria, RS, Brasil.

IIIPrograma de Pós-graduação em Ciência e Tecnologia dos Alimentos, UFSM, Santa Maria, RS, Brasil.

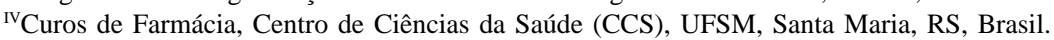

v Técnica de Laboratório, DTCA, CCR, UFSM, Santa Maria, Brasil. 
avestruz de aproximadamente $0,005 \mathrm{~kg} \mathrm{hab}^{-1} \mathrm{ano}^{-1}$, o que demonstra um aumento de $223 \%$ em relação ao ano anterior e uma franca possibilidade para expansão e introdução desse novo hábito alimentar na população.

Os produtos cárneos que utilizam como matéria-prima a carne de avestruz já são comercializados em países como Estados Unidos e Austrália, na forma de filés (steaks), salsicha, hambúrguer e jerked beef (JEFFEREY, 1999). FISHER et al. (2000) realizaram um estudo sobre processamento e características nutricionais de produtos com valor agregado de avestruz e concluíram que esses produtos são uma opção viável para serem utilizados na industrialização de carnes.

Os salames são definidos como produtos cárneos consistentes de uma mistura de carnes e partículas gordurosas, agentes de cura e condimentos que se classificam como produtos fermentados maturados e secos (FERNANDEZ et al., 2000) e sua produção é frequentemente conduzida em três etapas: formulação, fermentação e maturação/secagem (DEMEYER et al., 1986).

O objetivo deste trabalho foi avaliar os embutidos curados fermentados produzidos com diferentes concentrações de carne de avestruz, por meio de análises de $\mathrm{pH}$ (dias 0, 1, 2, 3, 4, 5, 6, 7, 14, 21 e 28), atividade de água (dias 0, 3, 7, 14, 21 e 28), quebra de peso e composição centesimal (dia 28), buscando a viabilidade de serem classificados de acordo com a legislação vigente e, assim, poderem ser lançados no mercado como uma alternativa de consumo.

\section{MATERIAL E MÉTODOS}

Os embutidos curados fermentados foram elaborados na Planta Piloto de Carnes do Departamento de Tecnologia e Ciência dos Alimentos da Universidade Federal de Santa Maria. A carne de avestruz (Struthio camelus) foi transportada até a Planta Piloto na forma congelada $\left(-18^{\circ} \mathrm{C}\right)$, em caixa térmica de poliestireno expandido. A matéria-prima foi descongelada por meio de descongelamento lento $\left(5^{\circ} \mathrm{C} 24 \mathrm{~h}^{-1}\right)$.

A formulação do produto foi constituída de quatro tratamentos, com diferentes porcentagens de carne suína e de carne de avestruz (tratamento controle - 76,68\% de carne suína; tratamento 1 - 19,08\% de carne de avestruz e 57,60\% de carne suína; tratamento 2 - 38,34\% de carne de avestruz e 38,34\% de carne suína e tratamento $3-57,60 \%$ de carne de avestruz e 19,08\% de carne suína). A quantidade dos demais ingredientes foram: toucinho suíno (19\%), cloreto de sódio (2,5\%), sais de cura ( $0,3 \%)$, fixador de cura $(0,2 \%)$, glicose $(0,4 \%)$, alho $(0,3 \%)$, sacarose $(0,4 \%)$, pimenta moída $(0,2 \%)$ e cultura starter Germinal ${ }^{\circledR}(0,02 \%)$, contendo os microorganismos Lactobacillus plantaram e Staphylococcus xylosus.

A fabricação do embutido foi conduzida segundo procedimento usual para o salame Tipo Italiano, segundo proposto por TERRA (1998). Foram elaborados $5 \mathrm{~kg}$ de cada tratamento, os quais foram embutidos em tripa artificial de colágeno não comestível com calibre de 60mm (Nutrim).

As peças, após o embutimento, foram mantidas em câmara de maturação (Menoncin ${ }^{\circledR}$, Erechim, Rio Grande do Sul), com umidade relativa, temperatura e velocidade do ar controladas, onde permaneceram por 28 dias, quando atingiram Atividade de Água (Aa) de 0,87.

A determinação do $\mathrm{pH}$ foi realizada segundo técnica proposta por TERRA e BRUM (1988), com amostras de $10 \mathrm{~g}$ homogeneizadas em $100 \mathrm{ml}$ de água destilada, e os valores foram medidos por meio de potenciômetro digital modelo DM20 (Digimed, São Paulo, Capital). A atividade de água foi medida por meio de aparelho modelo 400 CE (Testo GMBH \& Co., Lenzkirch, Alemanha). Para a determinação da quebra de peso, foi registrado o peso das peças do embutido no início e no final do processamento, por meio de balança digital modelo Exata 2SC (Toledo, São Bernardo do Campo, São Paulo).

As análises de composição centesimal foram realizadas no momento em que o processo de fabricação foi finalizado. Para tanto, foram utilizadas metodologias propostas por TERRA e BRUM (1988). A determinação da proteína foi feita por meio do método de micro Kjedahl, o qual consiste nas etapas de digestão, destilação e titulação. A determinação da umidade foi realizada por meio do método da estufa, que considera a perda de água e substâncias voláteis a $105^{\circ} \mathrm{C}$. A determinação da gordura foi realizada por meio do método do Butirômetro. A determinação das cinzas foi fundamentada na perda de peso quando o produto foi incinerado em mufla a $500^{\circ} \mathrm{C}$, com destruição da matéria orgânica.

O desenho experimental utilizado foi o inteiramente casualizado, com quatro níveis de carne de avestruz $(0 ; 19 ; 38,3 ; 57,6 \%)$, ou seja, quatro tratamentos sem repetições, totalizando quatro unidades experimentais. Os dados referentes à determinação do $\mathrm{pH}$, atividade de água e composição centesimal foram coletados em triplicata e submetidos à Análise de Variância (ANOVA), em nível de 5\% de significância, e comparados pelo teste de Tukey, também a 5\% de significância, sendo utilizado o software estatístico SPSS 8.0 (1998).

Ciência Rural, v.40, n.2, fev, 2010. 


\section{RESULTADOS E DISCUSSÃO}

Durante as fases do processamento dos embutidos fermentados, três grandes grupos de substâncias presentes são capazes de influenciar os valores de pH: os ácidos orgânicos oriundos da fermentação dos açúcares, os compostos básicos resultantes da proteólise gerada pelos microrganismos ou pelas próprias enzimas tissulares e os ácidos orgânicos procedentes das gorduras (CHAGAS, 1998).

Os embutidos, no dia do processamento, apresentaram valores médios de $\mathrm{pH}$ que variaram de 5,60 (tratamento controle) até 5,91 (tratamento 3), apresentando diferença significativa $(\mathrm{P}<0,05)$. Não há definição de valores de $\mathrm{pH}$ mínimo ou máximo permitido para salames (BRASIL, 2000), mas há um consenso para que esse valor, no produto final, não ultrapasse 5,4 (TERRA, 1998; TERRA et al., 2004), para garantir sua qualidade microbiológica. Notou-se uma queda acentuada desses números no $4^{\circ}$ dia de fabricação, para valores entre 4,58 e 4,78, em todos os tratamentos. $\mathrm{O}$ pH próximo ao ponto isoelétrico das proteínas $(5,3)$ é importante, pois reduz a capacidade de retenção de água, favorecendo a secagem e a perda de peso pelo produto cárneo fermentado, conferindo textura firme e fatiabilidade ao produto final (BUCKENHÜSKES, 1993).

As alterações no $\mathrm{pH}$ que ocorreram durante a fabricação dos embutidos elaborados neste trabalho estão de acordo com as expectativas. De acordo com TERRA et al. (2004), a partir do sétimo dia de fabricação, os valores de $\mathrm{pH}$ sofrem aumento em razão das reações de descarboxilação e da desaminação dos aminoácidos, liberando amônia no meio e alcalinizando-o. No entanto, o pH pode sofrer nova redução em razão da lipólise, que libera ácidos graxos no meio.
Estatisticamente, houve diferença significativa $(\mathrm{P}<0,05)$ para o $\mathrm{pH}$ entre os tratamentos em todos os dias analisados, com exceção do $6^{\circ}$ dia de fabricação. De acordo com a tabela 1 com exceção do tratamento 3 , houve uma elevação nos valores de $\mathrm{pH}$ a partir do sétimo dia de processamento. No entanto, esse aumento não foi suficiente para elevar os valores de $\mathrm{pH}$ a níveis adequados. Os valores obtidos neste trabalho foram mais ácidos do que os encontrados por TONETO (2007), que obteve médias entre 4,92 e 5,03 ao fim do processamento. Segundo TERRA (1998), uma acidificação excessiva pode ser resultado de processamento em altas temperaturas, adição exagerada de açúcar ou desenvolvimento de lactobacilos heterofermentativos que produzem ácidos mais fortes do que o ácido lático, como o acético e fórmico.

A atividade de água decresceu durante a fermentação e maturação dos embutidos. Inicialmente, de acordo com os resultados apresentados na tabela 2, os valores em todos os tratamentos foram de 0,99. Quando os produtos estavam finalizados, os valores encontrados foram entre 0,85 e 0,89. Esses valores são importantes, pois inibem o desenvolvimento de Pseudomonas, que é o principal agente de alteração da carne fresca, e consequentemente favorece o desenvolvimento de bactérias láticas e micrococacceae (ORDÓÑEZ et al., 2005). De acordo com o regulamento técnico de identidade e qualidade do salame (BRASIL, 2000), esses valores estão dentro daqueles determinados pela legislação, que deve ser no máximo de 0,92 para salame e 0,90 para salame tipo italiano.

A perda de água das peças de salame provocou uma quebra média de peso, variando de 30,22 a 38,48\%. Essa perda foi proporcional à quantidade de

Tabela 1 - Análise comparativa das médias, por tratamento, dos valores de pH pelo teste de Tukey.

\begin{tabular}{lllll}
\hline Dia & Controle $^{*}$ & Tratamento $1^{*}$ & Tratamento $2^{*}$ & Tratamento $^{*}$ \\
\hline Dia 0 & $5,60^{\mathrm{c}} \pm 0,00$ & $5,74^{\mathrm{b}} \pm-0,00$ & $5,77^{\mathrm{b}} \pm 0,03$ & $5,91^{\mathrm{a}} \pm 0,02$ \\
Dia 1 & $5,66^{\mathrm{c}} \pm 0,07$ & $5,80^{\mathrm{b}} \pm 0,01$ & $5,90^{\mathrm{ab}} \pm 0,00$ & $5,96^{\mathrm{a}} \pm 0,02$ \\
Dia 2 & $5,67^{\mathrm{b}} \pm 0,04$ & $5,81^{\mathrm{a}} \pm 0,02$ & $5,92^{\mathrm{a}} \pm 0,06$ & $5,87^{\mathrm{a}} \pm 0,02$ \\
Dia 3 & $5,21^{\mathrm{a}} \pm 0,02$ & $5,25^{\mathrm{a}} \pm 0,06$ & $5,24^{\mathrm{a}} \pm 0,09$ & $4,96^{\mathrm{b}} \pm 0,04$ \\
Dia 4 & $4,72^{\mathrm{a}} \pm 0,02$ & $4,71^{\mathrm{a}} \pm 0,04$ & $4,78^{\mathrm{a}} \pm 0,07$ & $4,58^{\mathrm{b}} \pm 0,01$ \\
Dia 5 & $4,49^{\mathrm{b}} \pm 0,01$ & $4,53^{\mathrm{ab}} \pm 0,04$ & $4,61^{\mathrm{a}} \pm 0,00$ & $4,52^{\mathrm{b}} \pm 0,02$ \\
Dia 6 & $4,38^{\mathrm{a}} \pm 0,05$ & $4,48^{\mathrm{a}} \pm 0,03$ & $4,44^{\mathrm{a}} \pm 0,08$ & $4,46^{\mathrm{a}} \pm 0,01$ \\
Dia 7 & $4,35^{\mathrm{a}} \pm 0,02$ & $4,42^{\mathrm{b}} \pm 0,02$ & $4,44^{\mathrm{b}} \pm 0,02$ & $4,57^{\mathrm{a}} \pm 0,02$ \\
Dia 14 & $4,32^{\mathrm{d}} \pm 0,01$ & $4,39^{\mathrm{c}} \pm 0,01$ & $4,45^{\mathrm{b}} \pm 0,02$ & $4,51^{\mathrm{a}} \pm 0,00$ \\
Dia 21 & $4,36^{\mathrm{c}} \pm 0,01$ & $4,50^{\mathrm{b}} \pm 0,02$ & $4,49^{\mathrm{b}} \pm 0,00$ & $4,57^{\mathrm{a}} \pm 0,00$ \\
Dia 28 & $4,38^{\mathrm{b}} \pm 0,02$ & $4,50^{\mathrm{a}} \pm 0,01$ & $4,49^{\mathrm{a}} \pm 0,02$ & $4,54^{\mathrm{a}} \pm 0,02$ \\
\hline
\end{tabular}

${ }^{a}$ Valores com sobrescritos diferentes em uma mesma linha são estatisticamente diferentes de acordo com o Teste de Tukey, a 5\%. ${ }^{*}$ Controle - 76,78\% de carne suína; Tratamento 1 - 19,08\% de carne de avestruz; Tratamento 2 - 38,34\%\% de carne de avestruz; Tratamento 3 $57,60 \%$ de carne de avestruz. 
Tabela 2 - Análise comparativa das médias, por tratamento, dos valores de Atividade de água (Aa) pelo Teste de Tukey e Quebra de Peso ao final do processamento (em \%).

\begin{tabular}{lcccc}
\hline & Controle $^{*}$ & Tratamento $^{*}$ & ${\text { Tratamento } 2^{*}}^{\text {Tratamento }^{*}}$ \\
\hline Dia 0 & $0,992^{\mathrm{a}} \pm 0,000$ & $0,990^{\mathrm{a}} \pm 0,002$ & $0,996^{\mathrm{a}} \pm 0,006$ & $0,986^{\mathrm{a}} \pm 0,017$ \\
Dia 3 & $0,979^{\mathrm{a}} \pm 0,009$ & $0,977^{\mathrm{a}} \pm 0,004$ & $0,976^{\mathrm{a}} \pm 0,003$ & $0,983^{\mathrm{a}} \pm 0,001$ \\
Dia 7 & $0,981^{\mathrm{a}} \pm 0,005$ & $0,970^{\mathrm{a}} \pm 0,005$ & $0,964^{\mathrm{b}} \pm 0,002$ & $0,972^{\mathrm{ab}} \pm 0,009$ \\
Dia 14 & $0,930^{\mathrm{b}} \pm 0,007$ & $0,948^{\mathrm{a}} \pm 0,003$ & $0,933^{\mathrm{b}} \pm 0,004$ & $0,949^{\mathrm{a}} \pm 0,004$ \\
Dia 21 & $0,925^{\mathrm{a}} \pm 0,009$ & $0,893^{\mathrm{c}} \pm 0,009$ & $0,920^{\mathrm{ab}} \pm 0,004$ & $0,905^{\mathrm{bc}} \pm 0,001$ \\
Dia 28 & $0,886^{\mathrm{a}} \pm 0,009$ & $0,846^{\mathrm{c}} \pm 0,011$ & $0,870^{\mathrm{ab}} \pm 0,003$ & 35,63 \\
Quebra de Peso & 30,22 & 33,69 & $38,032^{\mathrm{bc}} \pm 0,006$ & 38,48 \\
\hline
\end{tabular}

${ }^{a}$ Valores com sobrescritos diferentes em uma mesma linha são estatisticamente diferentes de acordo com o Teste de Tukey, a 5\%. ${ }^{*}$ Controle - 76,78\% de carne suína; Tratamento 1 - 19,08\% de carne de avestruz; Tratamento 2 - 38,34\% de carne de avestruz; Tratamento 3 - 57,60\% de carne de avestruz.

carne de avestruz, pelo fato de a carne dessa espécie possuir uma umidade superior ao da carne de outros animais, como bovinos e suínos (SALES \& HAYES, 1996), e ainda pelo fato de atingirem mais rapidamente o ponto isoelétrico das proteínas miofibrilares, reduzindo a capacidade de retenção de água e favorecendo sua secagem e perda de peso (BUCKENHÜSKES, 1993). Nota-se ainda que os produtos contendo carne de avestruz obtiveram valores de atividade de água (Tabela 2) e umidade (Tabela 3) inferiores aos valores do tratamento controle. CAMPAGNOL et al. (2007) obtiveram resultados semelhantes na fabricação de salame tipo italiano com 90\% de carne de ema, com valores de quebra de 33,10\% para peças feitas com tripa artificial e 40,46\% para embutidos com tripa natural.

Observando-se a tabela 3, nota-se que os valores de umidade total após a fabricação das peças ficaram entre 28,99 e 32,34\%, havendo diferença significativa $(\mathrm{P}<0,05)$ em todos os tratamentos estudados. Esses dados estão de acordo com a legislação vigente, que determina umidade em salame tipo italiano de no máximo 35\% (BRASIL, 2000). Os valores encontrados neste trabalho são semelhantes aos encontrados por CAMPAGNOL et al. (2007), em salame tipo italiano fabricado com carne de ema
(30,76\%), GRANER et al. (1983), em salames tipo italiano fabricados no Brasil (29,41\%), por CASIRAGHI et al. (1996), em salames tipo italiano e milano fabricados na Itália (36,25\%) e por SCHEID et al. (2003), que encontrou teores de umidade entre 23,4 e $37,5 \%$ ao final de 25 dias de processamento de salames tipo italiano com diferentes concentrações de cravo-daíndia.

Não foi observada diferença significativa $(\mathrm{P}<0,05)$ para os valores de proteína entre os tratamentos 1 e 2 . No entanto, esses valores foram significativamente maiores que os obtidos nos tratamentos 1 e 3 . Todos os resultados obtidos encontram-se dentro dos parâmetros de qualidade exigidos pela legislação, que devem ser superiores a 25\% (BRASIL, 2000), e variaram entre 27,24 e 29,47\%. Diferentemente de TONETO (2007), que obteve valores maiores de proteína em produtos com maior porcentagem de carne de ema, não foi possível notar neste trabalho relação entre os teores de proteína com a quantidade de carne de avestruz, já que o tratamento 3 apresentou valores inferiores aos tratamentos 1 e 2 . No entanto, esses valores foram semelhantes aos encontrados pelo autor anteriormente citado, que ficaram entre 29,89 e 31,03\%, e semelhante aos obtidos por CAMPAGNOL et al. (2007), que foram de $29,50 \%$

Tabela 3 - Análise comparativa das médias, por tratamento, dos valores de umidade, proteína, gordura e cinzas (em g\%) pelo teste de Tukey.

\begin{tabular}{lllll}
\hline & Controle $^{*}$ & Tratamento $^{*}$ & ${\text { Tratamento } 2^{*}}^{*}{\text { Tratamento } 3^{*}}^{*}$ & $31,40^{\mathrm{b}} \pm 0,26$ \\
Umidade & $32,34^{\mathrm{a}} \pm 0,39$ & $29,94^{\mathrm{c}} \pm 0,05$ & $28,99^{\mathrm{d}} \pm 0,20$ & $27,88^{\mathrm{b}} \pm 0,13$ \\
Proteína & $27,24^{\mathrm{c}} \pm 0,14$ & $29,09^{\mathrm{a}} \pm 0,15$ & $29,47^{\mathrm{a}} \pm 0,04$ & $35,32^{\mathrm{ab}} \pm 0,32$ \\
Gordura & $36,72^{\mathrm{a}} \pm 0,28$ & $34,14^{\mathrm{b}} \pm 0,69$ & $36,29^{\mathrm{ab}} \pm 0,03$ & $5,38^{\mathrm{a}} \pm 0,40$ \\
Cinzas & $5,57^{\mathrm{a}} \pm 0,29$ & $6,08^{\mathrm{a}} \pm 0,11$ & $5,80^{\mathrm{a}} \pm 0,11$ & \\
\hline
\end{tabular}

${ }^{a}$ Valores com sobrescritos diferentes em uma mesma linha são estatisticamente diferentes de acordo com o Teste de Tukey, a 5\%. ${ }^{*}$ Controle - 76,78\% de carne suína; Tratamento 1 - 19,08\% de carne de avestruz; Tratamento 2 - 38,34\% de carne de avestruz; Tratamento 3 - 57,60\% de carne de avestruz. 
com tripa artificial e 32,75\% com tripa natural. Os resultados encontrados neste trabalho foram superiores aos descritos por CAVENAGHI \& OLIVEIRA(1999) no estudo da influência de algumas características físicoquímicas e sensoriais na qualidade de salames fabricados no Brasil, em que os embutidos apresentaram em média 23,98\% de proteínas.

Os salames são produtos cárneos que apresentam elevado teor de gordura após a elaboração e podem aumentar até $40 \%$ esse valor durante a primeira semana, como resultado da desidratação e 40 a 50\% após quatro semanas da fabricação (WIRTH, 1988). Os resultados obtidos (Tabela 3) foram estatisticamente diferentes $(\mathrm{P}<0,05)$ entre o tratamento controle e o tratamento 1 e revelaram médias entre 34,14 e 36,72\%. Esses valores não se enquadram na legislação, que permite, no máximo, teor de 32\% de gordura (BRASIL, 2000). Assim como no trabalho de TONETO (2007), em que foram observados valores entre 19,67 e 23,53\%, a quantidade de gordura final nos produtos elaborados com carne de ratitas diminuiu à medida que a proporção de carne dessas aves aumentava na receita. COELHO (1999), avaliando a utilização de couro suíno no salame tipo italiano, encontrou teores de gordura finais entre 24,82 e $24,98 \%$, considerados baixos, que foram atribuídos à quantidade reduzida de toucinho utilizado na fabricação dos produtos. Como alternativa para a redução do teor de gordura final dos embutidos utilizando carne de avestruz, sugere-se a redução do teor de toucinho na formulação para 15 ou $10 \%$, com a finalidade de enquadrar esses produtos na legislação.

A análise das médias de cinzas (Tabela 3) evidencia que estatisticamente não houve diferenças significativas, e os resultados variaram entre 5,38 e $5,57 \%$, e a maior quantidade foi encontrada no tratamento com maior teor de carne de avestruz. TONETO (2007) também encontrou o maior teor final de cinzas nos embutidos com maior quantidade de carne de ema. Os valores encontrados por aquele autor (entre 5,91 e 6,37\%) e por CAMPAGNOL et al. (2007) (6,70\%) são semelhantes aos obtidos neste trabalho.

\section{CONCLUSÕES}

Os embutidos fabricados neste trabalho mostraram uma acidificação excessiva no final do processamento, aos 28 dias. A porcentagem de carne de avestruz influenciou sua composição centesimal, a qual, com exceção do teor de gordura, atendeu as especificações do Regulamento Técnico de Identidade e Qualidade do Ministério da Agricultura e Pecuária.

Em relação às formulações utilizadas, observa-se uma superioridade do tratamento 2 (38,34\% de carne de avestruz) em relação às outras, sendo então a formulação mais indicada quando se trabalha com embutidos curados fermentados que utilizam carne de avestruz como matéria-prima.

Neste experimento, foi demonstrada a viabilidade da utilização de carne de avestruz na fabricação de embutidos curados fermentados. Notase, ainda, a necessidade de novos estudos para que sejam obtidos produtos com valores de $\mathrm{pH}$ mais adequados e com formulações contendo teor reduzido de toucinho.

\section{AGRADECIMENTOS}

Os autores agradecem à Associação dos Estrutiocultores da Região Centro do Rio Grande do Sul, pela doação da carne de avestruz utilizada neste trabalho, tornando, assim, possível a sua realização.

\section{REFERÊNCIAS}

BANI, F.A. et al. Qualidade lipídica de carne de avestruz. Revista Nacional da Carne, n.361, p.23-26, 2007.

BRASIL. Instrução Normativa n. 22 de 31 de julho de 2000. Regulamentos técnicos de identidade e qualidade de salame tipo italiano. Publicado no Diário Oficial da União de 03 ago. 2000.

BUCKENHÜSKES, H.J. Selection criteria for lactic acid bacteria to be used as starter cultures for various food commodities. FEMS Microbiology Reviews, v.12, n.1-3, p.253-271, 1993.

CAMPAGNOL, P.C.B. et al. A carne de ema (Rhea americana) na fabricação de salame tipo italiano. Higiene Alimentar, v.21, n.148, p.60-63, 2007.

CASIRAGHI, E. et al. Quality attributes of milano salami and italian cured sausage. Journal of Agricultural and Food Chemistry, v.44, p.1248-1252, 1996.

CAVENAGHI, A.D.; OLIVEIRA, M.N. Influência de algumas características físico-químicas e sensoriais na qualidade de salame tipo italiano fabricado no Brasil. Revista Nacional da Carne, v.23, n.263, p.44-48, 1999.

CHAGAS, S.S. Redução do tempo de fabricação do salame tipo italiano. 1998. 99f. Dissertação (Mestrado em Ciência e Tecnologia dos Alimentos) - Programa de Pós-Graduação em Ciência e Tecnologia dos Alimentos. Universidade Federal de Santa Maria, RS.

COELHO, H.S. O couro suíno cozido na elaboração de salame tipo italiano. 1999. 89f. Dissertação (Mestrado em Ciência e Tecnologia dos Alimentos) - Universidade Federal de Santa Maria, RS.

DEMEYER, D.I. et al. Fermentation of meat: an integrated process. Food Chemical Biotechnology, v.41, p.131-140, 1986.

Ciência Rural, v.40, n.2, fev, 2010. 
FERNANDEZ, M. et al. Accelerated ripening of dry fermented sausages. Trands in Food Science \& Technology, v.11, p.201209, 2001. Disponível em: <http://dx.doi.org/10.1016/S09242244(00)00077-7>. Acesso em: 23 out. 2009. doi: 10.1016/ S0924-2244(00)00077-7.

FISHER, P. et al. Processing and nutritional characteristics of value added ostrich products. Meat Science, v.55, p.251-254, 2000. Disponível em: < http://dx.doi.org/10.1016/S03091740(99)00139-4>. Acesso em: 20 out. 2009. doi: 10.1016/ S0309-1740(99)00139-4.

GRANER, M. et al. Composição química de salames nacionais. Ciência e Tecnologia de Alimentos, v.3, n.1, p.48-57, 1983.

JEFFEREY, J. Ostrich production. Extension Veterinarian. Texas Agricultural Extension Service, The Texas A\&M University System, 1999. Disponível em: <http://gallus.tamu.ed/ ratite/ostrich.html>. Acesso em: 14 dez. 2008.

MUNIZ, L.R. Estatística censitária da estrutiocultura brasileira. In: ASSOCIAÇÃO DOS CRIADORES DE AVESTRUZES DO BRASIL. Anuário da estrutiocultura brasileira 2006/2007. São Paulo: ACAB, 2006. p.34-39.

MUNIZ, L.R. Estrutiocultura Brasileira: foco na exportação. In: SEMINÁRIO NORDESTINO DE PECUÁRIA, 12., 2008, Fortaleza, CE.. Disponível em: <http://www.pecnordeste.com.br/ pec2008/pdf/est/LuisRobsonMuniz.pdf>. Acesso: 15 jun. 2009.

ORDÓÑEZ, J.A. et al. Tecnologia de alimentos: alimentos de origem animal. Porto Alegre: Artmed, 2005. V.2, 279p.
SALES, J.; HAYES, J.P. Proximate, amino acid and mineral composition of ostrich meat. Food Chemistry, v.56, n.2, p.167-170, 1996. Disponível em: <http://dx.doi.org/10.1016/ 0308-8146(95)00201-4>. Acesso em: 17 out 2009. doi: 10.1016/0308-8146(95)00201-4.

SPSS Inc. SPSS Base 8.0. Applications Guide. Chicago: SPSS, 1998.

SCHEID, G.A. et al. Avaliação físico-química e sensorial de salame tipo italiano contendo diferentes concentrações de cravoda-índia (Eugenia caryophyllus). Ciência Agrotecnica, Ed. Especial, p.1576-1583, 2003.

TERRA, N.N.; BRUM, M.A.R. Carne e seus derivados: técnicas de controle de qualidade. São Paulo: Nobel, 1988. $121 \mathrm{p}$.

TERRA, N.N. Apontamentos de tecnologia de carnes. São Leopoldo: Unisinos, 1998. 216p.

TERRA, A.B.M. et al. Particularidades na fabricação de salame. São Paulo: Varela, 2004. 152p.

TONETO, E.R.L. Desenvolvimento de embutido curado fermentado de carne de ema (Rhea americana) associada à de suíno. 2007. 131f. Dissertação (Mestrado em Ciência e Tecnologia dos Alimentos) - Curso de Pós-graduação em Ciência e Tecnologia dos Alimentos, Universidade Federal de Santa Maria, RS.

WIRTH, F. Technologies for making fat-reduce meat products. What possibilities are there? Fleischwirtsch, v.68, n.9, p.11531156, 1988. 